\title{
The Role of Transbronchial Lung Biopsy in Diagnosing Pulmonary Mucormycosis in a Critical Care Unit
}

\author{
Yoonki Hong' and Jinkyeong Park ${ }^{2}$ \\ ${ }^{1}$ Department of Internal Medicine, Kangwon National University Hospital, Chuncheon; '2Department of Critical Care Medicine, Samsung Medical Center, \\ Sungkyunkwan University School of Medicine, Seoul, Korea
}

Background: Pulmonary mucormycosis (PM) is an emerging infectious disease and a life-threatening infection with high mortality. The clinical outcomes of PM have not improved significantly over the last decade because early diagnosis of PM is difficult and antifungal agents show limited activity. We evaluated the clinical manifestations of PM in a Korean tertiary hospital and identified the role of transbronchial lung biopsy (TBLB) in diagnosing PM in patients admitted to an intensive care unit.

Methods: The medical records of adult patients (aged 16 years and older) who met the criteria for proven or probable PM in a Korean tertiary hospital were retrospectively reviewed from January 2003 to December 2013. The clinical features, computed tomographic findings, diagnostic methods, treatment, and outcomes in patients with PM were evaluated.

Results: Of the nine patients, four were male. The median age was 64 years (range, 12 to 73 years). PM was proven and probable in seven and two cases, respectively. Computed tomography findings of PM were unilateral involvement in eight cases (89\%), consolidation in eight (89\%), ground glass opacity in four (44\%), and reverse halo sign in one (11\%). Six of nine cases (67\%) were diagnosed as PM from TBLB via portable bronchoscopy. There were no complications after TBLB. Mortality rate was 56\% (five of nine cases).

Conclusions: TBLB can be an easy and useful technique for diagnosing PM in the intensive care unit.

Key Words: biopsy; bronchoscopy; intensive care units; mucormycosis.

\section{Introduction}

Mucormycosis is an emerging infectious disease caused by fungi of the order Mucorales and represents the second leading cause of invasive fungal infection, following aspergillosis [1,2]. It is known to develop mainly in patients with diabetes, hematologic malignancy, organ transplantation, and those receiving immunosuppressive therapy [3]. The clinical presentation commonly results from involvement of rhinocerebral disease or pulmonary infection [4]. It was reported that Mucorales fungi most commonly affects the lungs in patients with hematological malignancies [5].

Pulmonary mucormycosis (PM) is a life-threatening infection with high mortality of over 70\% [6]. PM has recently emerged as patients with hematological malignancies or transplant patients have been on antifungal prophylaxis with Aspergillus-active but Mucorales-inactive agents [7]. Although its incidence was rare, reported to be 1.7 cases per million people/year by a population-based study [8], the incidence of PM is expected to increase because more individuals

\footnotetext{
Received on March 8, 2017 Accepted on April 24, 2017

Correspondence to: Jinkyeong Park, Department of Critical Care Medicine, Samsung Medical Center, Sungkyunkwan University School of Medicine, 81 Irwon-ro Gangnam-gu. Seoul 06351, Korea

Tel: +82-2-3410-3243, Fax: +82-2-3410-6956, E-mail: pjk3318@gmail.com

*No potential conflict of interest relevant to this article was reported.
}

(c) This is an Open Access article distributed under the terms of the Creative Commons Attribution Non-Commercial License (http://creativecommons.org/ licenses/by-nc/4.0/) which permits unrestricted non-commercial use, distribution, and reproduction in any medium, provided the original work is properly cited. Copyright (c) 2017 The Korean Society of Critical Care Medicine 
may be susceptible to invasive fungal infections resulting from either immunosuppressive disease or therapy with advances in modern medicine and because diagnosis of PM is difficult and underreported [4]. In these situations, more attention to PM may be needed in patients receiving intensive care, particularly those with immunocompromised status.

Unlike pulmonary aspergillosis, the clinical outcomes of PM have not improved significantly over the last decade, mainly because early diagnosis of PM is difficult and antifungal agents against Mucorales show limited activity [7]. Moreover, there are limited data differentiating computed tomography (CT) findings for PM from invasive pulmonary aspergillosis [9]. Although diagnostic methods for PM, such as fiber optic bronchoscopy with bronchoalveolar lavage or image-guided fine needle aspiration cytology, have been reported $[10,11]$, the accurate diagnosis of PM in patients in the intensive care unit remains a challenge.

At present, early and accurate diagnosis of PM is a very important issue for improving the therapeutic effect. We evaluated the clinical manifestations, imaging features, diagnosis, treatment, and prognosis of PM in a Korean tertiary hospital and identified the role of transbronchial lung biopsy (TBLB) in the diagnosis of PM in patients admitted to the intensive care unit.

\section{Materials and Methods}

This study was performed at the a tertiary-care teaching hospital in South Korea. The medical records of adult patients (aged 16 years and older) who met the criteria for proven or probable PM were retrospectively reviewed from January 2003 to December 2013.

Proven and probable PM were defined as described by the revised criteria of the European Organization for Research and Treatment of Cancer/Mycosis Study Group [12]. Proven PM was defined by histological evidence of non-septate, right-angle branching filamentous fungi invading tissue plus culture positive for Mucorales species from pulmonary tissue or immunohistochemical staining positive for anti-Rhizopus arrhizus monoclonal antibody (LSBio, Seattle, WA, USA). Probable PM was defined as the presence of host factors plus one or more clinical indications. The host factors included uncontrolled diabetes, hematological malignancy, organ transplantation, and immunosuppressive therapy. Furthermore, the clinical indications included Mucorales growth in sputum or bronchoalveolar lavage fluid culture or CT finding such as dense well-circumscribed lesions with or without a halo sign or an air-crescent sign or cavity.

TBLB was performed on the lobe in which CT findings suspected involvement of PM in the intensive care unit via portable bronchoscopy. A chest X-ray and medical records after TBLB were reviewed to exclude pneumothorax and bleeding.

Evaluation in each patient included (1) an underlying condition of immunocompromised state, (2) clinical presentations, (3) CT findings (unilateral or bilateral involvement, nodule, mass, consolidation, cavitation, ground glass opacity, air-fluid level, pleural effusion, and halo sign), (4) cytomorphological features and a final diagnosis of specimen, (5) complications following the diagnostic procedure, and (6) treatment response.

The data are presented as medians (range) for continuous variables and as percentages for discrete variables. These variables were analyzed by using simple descriptive statistics.

All patients gave their written informed consent for bronchoscopy and TBLB. Institutional review board of Samsung Medical Center approved the analyses of the clinical and TBLB data. Ethical approval for this study was exempted by the institutional review board (SMC 2017-02-023-002).

\section{Results}

Characteristics of PM patients and their underlying diseases are summarized in Table 1. Of the nine patients, four were male. The median age was 64 years (range, 12 
Table 1. Baseline characteristics and underlying disease of patients with pulmonary mucormycosis

\begin{tabular}{lc}
\multicolumn{1}{c}{ Characteristic } & Value \\
\hline Age (yr) & $64(12-73)$ \\
Sex (M/F) & $4 / 5$ \\
Underlying disease & \\
$\quad$ Malignancy & 9 \\
$\quad$ Hematologic malignancy & $7(77.8)$ \\
$\quad$ Solid cancer & $2(22.2)$ \\
$\quad$ Diabetes mellitus & 3 \\
Proven/probable pulmonary mucormycosis & $7 / 2$ \\
Diagnostic method & \\
Transbronchial lung biopsy & 6 \\
Surgical biopsy & 2 \\
Percutaneous needle aspiration & 1 \\
\hline
\end{tabular}

Values are presented as mean (range), number, or number (\%).

Table 2. CT findings of pulmonary mucormycosis

\begin{tabular}{lc}
\hline Variable & No. (\%) \\
\hline Unilateral/bilateral & $8(89) / 1(11)$ \\
Nodule & $2(22)$ \\
Consolidation & $8(89)$ \\
Mass & $3(33)$ \\
Cavitation & $3(33)$ \\
Ground glass opacity & $4(44)$ \\
Air-fluid level & 0 \\
Pleural effusion & $2(22)$ \\
Reverse halo sign & $1(11)$ \\
Suggestion of pulmonary infection by radiologist on CT & \\
findings & \\
Bacterial pneumonia & $4(44)$ \\
Atypical pneumonia & $1(11)$ \\
Actinomycosis & $1(11)$ \\
Fungal infection & $3(33)$ \\
\hline
\end{tabular}

CT: computed tomography.

to 73 years). The underlying diseases at diagnosis of PM were hematologic malignancy (seven cases) and solid cancer (two cases). Three patients had diabetes mellitus, and nine patients had received chemotherapy prior to diagnosis of PM. PM was proven and probable in seven and two cases, respectively.

CT findings of PM are summarized in Table 2. Unilateral involvement was shown in eight cases $(89 \%)$, con- solidation in eight cases (89\%), and ground glass opacity in four cases. However, reverse halo sign was only shown in one case (11\%). Fungal infections, such as invasive pulmonary aspergillosis or PM, suspected on CT by radiologists were present in three cases (33\%). Four cases $(44 \%)$ were suspected to have bacterial pneumonia on $\mathrm{CT}$.

The mortality rate was $56 \%$ (five of nine cases) (Table 3). Pathologic findings of lung specimens by each diagnostic procedure showed seven cases $(78 \%)$ compatible with mucormycosis and two cases (22\%) suggesting fungal infections of invasive aspergillosis or mucormycosis. The median treatment period was 35 days (range, 3 to 154 days).

Six of nine cases $(67 \%)$ were diagnosed with PM from TBLB via portable bronchoscopy. There were no complications such as pneumothorax and hemorrhage after TBLB on chest radiography (CXR) and medical records. One case was diagnosed by percutaneous lung aspiration/ biopsy, and two cases were diagnosed by surgical biopsy and resection. The condition of the two PM cases receiving surgical resection improved following administration of antifungal agents of liposomal amphotericin. None of the six cases diagnosed by TBLB could receive surgical treatment due to poor general condition, only one case $(16.7 \%)$ improved after medical treatment.

\section{Discussion}

The present study shows that PM is associated with high mortality rates, which might be improved in cases receiving surgical treatment. Moreover, TBLB might be an early and feasible tool for diagnosing PM in suspected critical patients.

Little is known about the clinical presentations of PM. Most of the data showing clinical features of mucormycosis, including PM, has originated from case series and small-sized studies on specific patient groups such as patients with hematologic malignancies [5,10]. Known clinical manifestations of PM are summarized as fol- 
Table 3. Treatment and outcomes of pulmonary mucormycosis

\begin{tabular}{ccccccccccc}
\hline Case & Age (yr) & Sex & $\begin{array}{c}\text { Underlying } \\
\text { disease }\end{array}$ & ANC & $\begin{array}{c}\text { Diagnostic } \\
\text { method }\end{array}$ & CT diagnosis & Pathology & Drug & Period (d) $^{\mathbf{a}}$ & Outcome \\
\hline 1 & 69 & F & AML & 290 & TBLB & BP or AP & R/O & Amphotericin B & 3 & Death \\
2 & 17 & M & NHL & 160 & TBLB & Fungal infection & C/W & Amphotericin B & 50 & Alive \\
3 & 73 & F & PNH & 10,720 & TBLB & BP & C/W & L-amphotericin & 9 & Death \\
4 & 55 & M & AML & 1,440 & TBLB & BP & C/W & Amphotericin & 21 & Death \\
5 & 66 & M & Multiple cancer & 2,920 & PCNA & Actinomycosis & R/O & Itraconazole & 84 & Alive \\
6 & 12 & M & LCH & 1,460 & VATs & Fungal infection & C/W & L-amphotericin & 154 & Alive \\
7 & 70 & F & RCC & 6,060 & TBLB & BP & C/W & Amphotericin B & 15 & Death \\
8 & 64 & F & AML & 1,980 & TBLB & BP & C/W & L-amphotericin & 35 & Death \\
9 & 14 & F & AML & 300 & VATs & Fungal infection & C/W & L-amphotericin & 144 & Alive \\
\hline
\end{tabular}

ANC: absolute neutrophil count at diagnosis of pulmonary mucormycosis; CT: computed tomography; AML: acute myeloid leukemia; TBLB: transbronchial lung biopsy; BP: bacterial pneumonia; AP: atypical pneumonia; R/O: further need differentiate between aspergillosis and mucormycosis; NHL: non-Hodgkin's lymphoma; C/W: compatible with mucormycosis; PNH: paroxysmal nocturnal hemoglobinuria; L-amphotericin: liposomal amphotericin; PCNA: percutaneous needle aspiration; LCH: langerhans cell histiocytosis; VATs: video assisted thoracoscopic surgery; RCC: renal cell carcinoma.

${ }^{\text {TT} T r e a t m e n t ~ p e r i o d s ~ w i t h ~ a n t i f u n g a l ~ a g e n t s . ~}$

lows: (1) underlying host risk factors were neutropenia, induction chemotherapy, hematopoietic stem cell transplantation with graft-versus-host disease, and lung transplantation [7]; (2) the pathogenesis of the disease state was hyphal invasion of pulmonary blood vessels, which could result in hemorrhage, thrombosis, ischemia, and infarction of distal tissue [7,13]; (3) clinical characteristics were prolonged high-grade fever $\left(>38^{\circ} \mathrm{C}\right)$, nonproductive cough, airway obstruction from endobronchial or tracheal lesions, massive hemoptysis; and (4) the mortality rate was $66 \%$ or higher, depending on the level of immunosuppression $[6,14]$. To date, however, there is no clinical history specific for the diagnosis of PM.

It is typically difficult to suspect PM and make a diagnosis early because PM is characterized by non-specific clinical features and radiologic findings, a low culturepositive rate, and a lack of biologic markers [4]. There were several reports that $\mathrm{CT}$ findings useful for the diagnosis of PM were progressive, homogeneous lobar or multilobar consolidation; nodules or mass-like or wedgeshaped consolidation; and a halo sign of ground glass opacity surrounding a pulmonary nodule $[15,16]$. On the other hand, Jung et al. [9] showed that the reverse halo sign helps the clinician to suspect PM and differentiate it from invasive pulmonary aspergillosis. A fungal culture of specimens obtained from a sputum culture, bronchoalveolar lavage culture, or percutaneous needle aspirate may be more helpful in the diagnosis of PM. Although the diagnosis of mucormycosis was recommended as proven, probable, or possible according to a host factor, a clinical criterion, and a mycological criterion [12], a definitive diagnosis of PM requires histologic identification of the organism, seen as mucoraceous hyphae in affected lung tissues [7,17]. Bronchoalveolar lavage, percutaneous needle aspiration, open lung biopsy, and pleural fluid culture are considered as diagnostic techniques to determine the affected lung. Of these techniques, the risk of surgery and the low incidence of pleural involvement limits the diagnostic value of open lung biopsy and pleural fluid culture. Moreover, it was recommended that isolation of the organism only from sputum or bronchial aspirate from bronchoalveolar lavage was not sufficient for a definite diagnosis [12,17], although it had been reported that analysis of an adequate bronchoalveolar lavage specimen from fiber optic bronchoscopy was a useful diagnostic method [10]. Recently, Sharma et al. [11] evaluated the role of fine needle aspiration cytology in the diagnosis of pulmonary infections in 42 immunocompromised patients and showed that it was a relatively reliable, safe, and rapid method of diagnosing pulmonary 
infection, including PM, in immunocompromised patients. In this study, we showed that TBLB might also be a relatively reliable and safe method of diagnosing PM in immunocompromised patients. Especially in the intensive care unit, TBLB could be a useful tool for diagnosing PM in suspected patients because TBLB is easier and more feasible than conducting portable bronchoscopy at the bedside.

Treatment of PM is complicated because the organisms, Mucorales, are inherently resistant to antifungal agents $[18,19]$, and PM demonstrates rapid clinical progression [7]. There are also limited data for antifungal susceptibility and available results of minimum inhibitory concentration testing. Moreover, it is difficult to use empirical antifungal agents for suspected patients with invasive fungal infections because Mucorales may express increased virulence following exposure to voriconazole [20], a widely used treatment for invasive pulmonary aspergillosis, which has a clinical presentation similar to that of PM. Therefore, at present, an early and accurate diagnosis is paramount in the treatment of PM. The main treatment strategy for PM is immediate surgical resection of the infected lung, such as wedge resection, lobectomy, or pneumonectomy, in combination with antifungal agents. These treatments have been associated with lower mortality rates in many reported cases [21-23]. Additionally, for medical therapy for PM, the only recommended antifungal agents are members of the polyene class, including amphotericin B deoxycholate and its lipid derivatives. Triazoles, posaconazole, isavuconazole, echinocandins, and combination therapy could be also considered [4]. In our study, two patients receiving surgical resection were alive after treatment. However, it may be considered that patients in good condition are more likely to receive surgery.

This report has limitations due to its retrospective and non-controlled nature. Long-term observational and interventional controlled studies are needed for more advanced knowledge of PM. Moreover, clinicians should be concerned with complications of TBLB, though there were no cases of pneumothorax nor hemorrhage in this study.

PM is an emerging invasive infection and is often fatal, and its successful treatment relies on a timely diagnosis. TBLB can be an easy and useful technique for diagnosing PM in the intensive care unit.

\section{ORCID}

Yoonki Hong http://orcid.org/0000-0002-1607-6777

Jinkyeong Park http://orcid.org/0000-0002-8833-9062

\section{References}

1. Garcia-Hermoso D, Dannaoui E, Lortholary O, Dromer F. Agents of systemic and subcutaneous mucormycosis and entomophthoromycosis. In: Versalovic J, Carroll KC, Funke G, Jorgensen JH, Landry ML, Warnock DW, editors. Manual of clinical microbiology. 10th ed. Washington, DC: ASM Press; 2011. p. 1880-901.

2. Kontoyiannis DP, Lionakis MS, Lewis RE, Chamilos G, Healy M, Perego C, et al. Zygomycosis in a tertiary-care cancer center in the era of Aspergillus-active antifungal therapy: a case-control observational study of 27 recent cases. J Infect Dis 2005;191:1350-60.

3. Ibrahim AS, Spellberg B, Walsh TJ, Kontoyiannis DP. Pathogenesis of mucormycosis. Clin Infect Dis 2012;54 Suppl 1:S16-22.

4. Riley TT, Muzny CA, Swiatlo E, Legendre DP. Breaking the mold: a review of mucormycosis and current pharmacological treatment options. Ann Pharmacother 2016;50:747-57.

5. Pagano L, Offidani M, Fianchi L, Nosari A, Candoni A, Picardi M, et al. Mucormycosis in hematologic patients. Haematologica 2004;89:207-14.

6. Roden MM, Zaoutis TE, Buchanan WL, Knudsen TA, Sarkisova TA, Schaufele RL, et al. Epidemiology and outcome of zygomycosis: a review of 929 reported cases. Clin Infect Dis 2005;41:634-53. 
7. Hamilos G, Samonis G, Kontoyiannis DP. Pulmonary mucormycosis. Semin Respir Crit Care Med 2011;32:693-702.

8. Rees JR, Pinner RW, Hajjeh RA, Brandt ME, Reingold AL. The epidemiological features of invasive mycotic infections in the San Francisco Bay area, 1992-1993: results of population-based laboratory active surveillance. Clin Infect Dis 1998;27:1138-47.

9. Jung J, Kim MY, Lee HJ, Park YS, Lee SO, Choi $\mathrm{SH}$, et al. Comparison of computed tomographic findings in pulmonary mucormycosis and invasive pulmonary aspergillosis. Clin Microbiol Infect 2015;21:684.e11-8.

10. al-Abbadi MA, Russo K, Wilkinson EJ. Pulmonary mucormycosis diagnosed by bronchoalveolar lavage: a case report and review of the literature. Pediatr Pulmonol 1997;23:222-5.

11. Sharma S, Gupta P, Gupta N, Lal A, Behera D, Rajwanshi A. Pulmonary infections in immunocompromised patients: the role of image-guided fine needle aspiration cytology. Cytopathology 2017;28:46-54 .

12. De Pauw B, Walsh TJ, Donnelly JP, Stevens DA, Edwards JE, Calandra T, et al. Revised definitions of invasive fungal disease from the European organization for Research and Treatment of Cancer/Invasive Fungal Infections Cooperative Group and the National Institute of Allergy and Infectious Diseases Mycoses Study Group (EORTC/MSG) Consensus Group. Clin Infect Dis 2008;46:1813-21.

13. Kim YI, Kang HC, Lee HS, Choi JS, Seo KH, Kim $\mathrm{YH}$, et al. Invasive pulmonary mucormycosis with concomitant lung cancer presented with massive hemoptysis by huge pseudoaneurysm of pulmonary artery. Ann Thorac Surg 2014;98:1832-5.

14. Neto FM, Camargo PC, Costa AN, Teixeira RH, Carraro RM, Afonso JE Jr, et al. Fungal infection by Mucorales order in lung transplantation: 4 case reports. Transplant Proc 2014;46:1849-51.

15. McAdams HP, Rosado de Christenson M, Strollo DC, Patz EF Jr. Pulmonary mucormycosis: radio- logic findings in 32 cases. AJR Am J Roentgenol 1997; 168:1541-8

16. Jamadar DA, Kazerooni EA, Daly BD, White CS, Gross BH. Pulmonary zygomycosis: CT appearance. J Comput Assist Tomogr 1995;19:733-8.

17. Walsh TJ, Gamaletsou MN, McGinnis MR, Hayden RT, Kontoyiannis DP. Early clinical and laboratory diagnosis of invasive pulmonary, extrapulmonary, and disseminated mucormycosis (zygomycosis). Clin Infect Dis 2012;54 Suppl 1:S55-60.

18. Almyroudis NG, Sutton DA, Fothergill AW, Rinaldi MG, Kusne S. In vitro susceptibilities of 217 clinical isolates of zygomycetes to conventional and new antifungal agents. Antimicrob Agents Chemother 2007;51:2587-90.

19. Drogari-Apiranthitou M, Mantopoulou FD, Skiada A, Kanioura L, Grammatikou M, Vrioni G, et al. In vitro antifungal susceptibility of filamentous fungi causing rare infections: synergy testing of amphotericin $\mathrm{B}$, posaconazole and anidulafungin in pairs. J Antimicrob Chemother 2012;67:1937-40.

20. Lamaris GA, Ben-Ami R, Lewis RE, Chamilos G, Samonis G, Kontoyiannis DP. Increased virulence of Zygomycetes organisms following exposure to voriconazole: a study involving fly and murine models of zygomycosis. J Infect Dis 2009;199:1399-406.

21. Schneidawind D, Nann D, Vogel W, Faul C, Fend F, Horger M, et al. Allogeneic hematopoietic cell transplantation in patients with acute myeloid leukemia and pulmonary mucormycosis. Transpl Infect Dis 2012;14:E166-72.

22. Fitzpatrick MC, Carter BW. Pulmonary mucormycosis complicating cutaneous blastic plasmacytoid dendritic cell neoplasm. Proc (Bayl Univ Med Cent) 2012;25:287-8.

23. Serio B, Rosamilio R, Giudice V, Zeppa P, Esposito $\mathrm{S}$, Fontana R, et al. Successful management of pulmonary mucormycosis with liposomal amphotericin B and surgery treatment: a case report. Infez Med 2012;20 Suppl 2:43-7. 